\title{
Improving the Bit Error Rate Performance of Free Space Optical Communication due to Atmospheric Turbulence Effect using New Double Multiple-Input Multiple-Output Technique
}

\author{
A K Rahman \\ Department of Electrical and Electronics \\ Universiti Malaysia Sarawak \\ Kuching, Malaysia \\ karahman@unimas.my
}

\author{
A L Tom \\ Department of Electrical and Electronics \\ Universiti Malaysia Sarawak \\ Kuching, Malaysia \\ amlionyy@gmail.com
}

\begin{abstract}
This paper focus on mitigating the atmospheric turbulence effect in free space optical (FSO) communications by using double multiple input multiple output (DMIMO) technique. This technique is an enhancement of the conventional multiple input multiple output (MIMO) technique in improving the performance of FSO system. The combination of double MIMO with differential detection mode at the receiver resulting in superior modulation against the turbulence especially in reducing the scintillation index, enhance the power received and improve the threshold signal level. The analysis result shows that under strong turbulence conditions, the bit error rate can be improved by 20 percent in terms of free space optical distance propagation.
\end{abstract}

Keywords - bit error rate, free space optical communications, atmospheric turbulence, multiple input multiple output.

\section{INTRODUCTION}

Free space optical communications (FSOC) is an optical wireless communication technology that uses light between two points to transmit data. Due to its large bandwidth potential, unlicensed spectrum, excellent security, and inexpensive setup, FSOC technology became popular. New networking technologies such as high speed internet, videoconferencing, live streaming etc. have raised the demands of higher data rates and require mobility and versatility in telecommunication networks. The demands of higher data rates for various telecommunications and multimedia services have resulted to spectrum congestion from RF to optical carrier [1]-[3].

While FSOC system has many appealing features, due to the degrading effects of atmospheric turbulence-induced fading it has somewhat disappointing performance for long links. There are three significant challenges to be tackled in this regard when using it as a communication channel; absorption, scattering, and turbulence [4]. We are focussing on atmospheric turbulence in this paper. One significant disadvantage faced by FSOC is the effect on device efficiency of turbulence-induced irradiance fluctuation. The atmospheric turbulence is generated by spatial and temporal random fluctuations of refractive index caused by variations in temperature, pressure, and wind along the channel's optical direction of propagation [5].

The performance of wireless systems can be enhanced through spatial diversity, particularly the multiple input multiple output (MIMO) technique. MIMO has the potential to dramatically improve the efficiency of wireless system as they can exploit the diversity in spatial and time domains. MIMO systems improve the spectral efficiency by multiplexing data on separate parallel channels without incurring any cost in terms of bandwidth or power. A MIMO system achieves multiplexing and diversity gains as a strong function of the channel characteristics, which in turn depends on the scattering environment and the configuration of the array deployed at the transmitter and the receiver. To further improve the performance of the FSO system, and to mitigate the impact atmospheric turbulence, this new approach of conventional MIMO, namely DMIMO, is proposed. DMIMO is a system where the FSO is employed with doubles of the number of transmitters and receivers than the conventional MIMO.

The remaining part of the paper is organized as follows. The system model and channel modelling are describes in Section 2 and Section 3, respectively. Section 4 gives the mathematical analysis of signal-to-noise ratio (SNR) and bit error rate (BER) of the DMIMO FSO system with intensity modulation/direct-detection (IM/DD) with on-off keying (OOK). The results are discussed and compared in in Section 5. Section 6 concludes the paper.

\section{SYSTEM DESIGN MODEL}

This Double Multiple Input Multiple Output (DMIMO) employs multiple number of $M$ transmitter lasers and multiple number of $N$ receiver photodetectors, transmitted and received over discrete time ergodic channel with additive white Gaussian noise (AWGN). On-Off Keying (OOK) is used to modulate the bits of information. The received signal at the $n$ th receiver aperture is given by

$$
r_{n}=x \eta \sum_{m=1}^{M} \frac{I_{m n}}{2 M N}+z_{n}
$$

where $x \in[0,1]$ represents the information bits, $\eta$ is the optical-to-electrical conversion coefficient, $z_{n}$ is the AWGN with zero mean and variance $N_{0} / 2$ and $I_{m n}$ denotes the normalized irradiance of the link between $m$ th laser and $n$th aperture. In equation (1) the scaling factor $M N$ appears to ensure the comparison between single input single output (SISO), MIMO, and DMIMO FSO system.

\section{A. Transmitter}

The transmitter is made of four elements. First is the pseudo-random bit sequence generator (PRBS). This 